\title{
AN APPROACH TO DAMAGE DETECTION IN THE AIRCRAFT STRUCTURE WITH THE USE OF INTEGRATED SENSORS - THE SYMOST PROJECT
}

\author{
Krzysztof Dragan ${ }^{1,2}$ \\ Michał Dziendzikowski ${ }^{1}$ \\ Andrzej Leski ${ }^{1}$ \\ Ziemowit Dworakowski ${ }^{1}$ \\ Tadeusz $\mathbf{U h l}^{2}$
}

Air Force Institute of Technology, Warsaw, Poland

AGH University of Technology, Krakow, Poland

\begin{abstract}
This paper presents an approach to damage growth monitoring and early damage detection in the structure of PZL - 130 ORLIK TC II turbo-prop military trainer aft using the statistical models elaborated by the Polish Air Force Institute of Technology (AFIT) and the network of the sensors attached to the structure. Drawing on the previous experiences of the AFIT and AGH in structural health monitoring, the present research will deploy an array of the PZT sensors in the structure of the PZL -130 Orlik TC II aircraft. The aircraft has just started Full Scale Fatigue Test (FSFT) that will continue up to 2013. The FSFT of the structure is necessary as a consequence of the structure modification and the change of the maintenance system - the transition to Condition Based Maintenance. In this paper, a novel approach to the monitoring of the aircraft hot-spots will be presented. Special attention will be paid to the preliminary results of the statistical models that provide an automated tool to infer about the presence of damage and its size. In particular, the effectiveness of the selected signal characteristics will be assessed using dimensional reduction methods (PCA) and the so-called averaged damage indices will be delivered. Moreover, the results of the signal classification based on the neural network will be presented alongside the numerical model of the wave propagation. The work contains selected information about the project scope and the results achieved at the preliminary stage of the project.
\end{abstract}

Keywords: averaged damage index, Full Scale Fatigue Test, damage growth monitoring

\section{INTRODUCTION}

The problems associated with monitoring of health of aircraft structures throughout the operational phase (operation and maintenance) are very complex and complicated. The spectrum of variable loads that affect a structure depends first and foremost on the way the aircraft is operated. Hence, it is impossible to precisely design the aircraft life before it enters the operational use. This paper presents an approach to developing an integrated monitoring system validated for the operational phase. The aircraft used in the research is PZL ORLIK TC II, a turbo propeller training aircraft for the armed forces. The aircraft was designed in Poland in the PZL WSK Warszawa Okecie and at present is manufactured at the PZL EADS Okecie in Warsaw. The main 
use of the aircraft is for training military pilots in the preliminary training phase. The aircraft has just started Full Scale Fatigue Test (FSFT) that will continue up to 2013. The FSFT of the structure is necessary as a conseqience of the structure modification and the change of the maintenance system (from the Safe - Life to CBM). The AFIT's contribution to the FSFT was the NDI programme including health monitoring techniques and the know-how. In particular, an alternative SHM system based on PZT sensors will be designed and verified during FSFT to support or possibly partially replace scheduled inspections in the future.

This SHM system should follow the international standards for such systems known as the SHM paradigm [1], which means that the system is organized in the hierarchical structure:

- damage identification and localization;

- damage classification and size quantification;

- residual life estimation.

In the project, multiple tasks are oriented to achieve the highest possible technical readiness of the system (in the operational way of thinking). However, there are still a few challenges associated with the SHM system design such as [2]:

- utilizing formal methods for designing sensing and data processing systems;

- correlating damage indications with remaining structural capabilities;

- minimizing false calls resulting from benign changes in operation and boundary conditions;

- addressing durability of on-board hardware.

This work focuses only on selected applications of such a system consistent with the SHM paradigm and associated with the quantification of the damage size. A statistical approach based on averaged damage indices and supervised learning (SL) techniques will be highlighted. The paper will present an approach to classification of the damage indices from time signals obtained from the PZT sensors of the guided waves propagation. That will include: damage indices selection, classification models, self diagnosis application and SL techniques.

\section{STRUCTURAL HEALTH MONITORING}

Depending on their source and the geometry of the structure under consideration, elastic waves can propagate a significant distance. They are also sensitive to local structure discontinuities and deformations providing a tool to detect local damage of large aerospace structures. One of the major obstacle in a direct application of this method is the complexity of signals excited in real structures. Reliable SHM systems should therefore provide a different type of damage assessment to allow for cross-validated evaluation of the structure:

- qualitative data - damage presence in a given network cell, its type and the order of magnitude;

- quantitative data - exact location and size of damage.

Basic information concerning the health of a structure can be provided by the so-called damage indices (DI's). Denoting as $f_{g s}$ the signal generated in the transducer $g$ and received in the sensor $s$ and denoting as $f_{g s, b}$ its baseline, certain basic DI's can be defined using the following simple signal characteristics: 


$$
\begin{gathered}
L^{1} \text { symmetric characteristic }-D I_{1}(g, s)=\frac{\left|\int\right| f_{g s}|-| f_{g s, b}|d t|}{\int\left|f_{g s, b}\right| d t}, \\
L^{2} \text { symmetric characteristic }-D I_{2}(g, s)=\frac{\int\left(f_{g s}\right)^{2}-\left(f_{g s, b}\right)^{2} d t}{\int\left(f_{g s, b}\right)^{2} d t}, \\
\text { correlation with the baseline }-D I_{3}(g, s):=\operatorname{cor}(g, s) .
\end{gathered}
$$

Similar DI's can be obtained using the Fourier filtered signals, their envelopes or other signal transformations. These simple signal characteristics can be useful for qualitative assessment due to their small volatility. They have positive sign irrespectively of the amount of energy received by the given sensor, which is an important property when averaging.

There exist remarkable examples of applications of analogous DI's to determine localization of damage [8-10]. In these methods two stage algorithms are used. First, for each sensing path $g \rightarrow s$, i.e. a signal received in the sensor $s$ originated from the generator $g$, the structure is quantified into a damaged or undamaged state. This quantitative assessment can be performed using a certain threshold level of one or multiple DI's. If the structure is considered as damaged, the probable density of the damage localization in a given network cell is calculated. This density depends on DI's values and a properly defined distance of a given network point from the sensing path $g \rightarrow s$. Finally, the joint probability for a damage localization is provided using probability maps obtained for all possible sensing paths in the network cell.

There are several limitations of the described SHM method. In particular, in order to obtain an accurate damage location probability map it is necessary to consider sensing paths for many transducers, which affects the computational and system implementation costs. Furthermore, improper functioning of a single sensor in the network decreases the number of reliable sensing paths, which can disturb this probability density. Another obstacle in this approach is the system sensitivity adjustment. In complex structures that contain many wave reflectors, e.g. edges, joints, welds, rivets, etc., a resulting map for sensitive algorithms can be noised[11], whereas weakening susceptibility of sensing paths causes risk of missing damage. However, the main disadvantage of that method is the difficulty in estimating the damage size. Since damage indices $D I_{j}(g, s)$ used for structure quantification in these algorithms depend strongly on damage localization with respect to a given sensing path $g \rightarrow s$, it is difficult to use regression or classification models in estimating the damage size.

\subsection{Damage Growth Monitoring}

Reliable damage size assessment requires developing methods independent of or adjusted to the damage location. Therefore, for a given damage index $D I_{j}(g, s)$ the averaged damage index can be defined $[12,13]$ as:

$$
A D I_{j}:=\frac{1}{G(S-1)} \sum_{\substack{g, s: \\ g \neq s}} D I_{j}(g, s),
$$

where $G=S$ is the number of transducers in the sensor network cell. Averaged damage indices (ADI's) are less dependent on the damage localization, i.e. they are invariant with respect to sensors permutation, which makes them better suited for damage size estimation. These indices can be defined in the case of improper functioning of several transducers in the network. Moreover, they contain joint information from all sensors, which should improve possibility of small damage detection. It is therefore important to use constant sign DI's in their definition to avoid cancellation of factors. 
The efficiency of the proposed signal characteristics can be evaluated using ADI's and Principal Component Analysis (PCA). PCA is an effective feature extraction algorithm and can be used in predictive models development. This method was frequently used for the SHM purposes [14-18]. Subsequent principal components $\lambda_{i}$ are linear combinations of all ADI's used:

$$
\lambda_{i}=\sum_{j=1}^{D} d_{i}^{j} A D I_{j}, \quad i=1, \ldots, D,
$$

where $D$ is the number of indices considered. The coefficients $d_{i}^{j}$ that are components of normalized vectors satisfy

$$
\sum_{i=j}^{D}\left(d_{i}^{j}\right)^{2}=1, \quad i=1, \ldots, D .
$$

Typically, values of these coefficients corresponding to different characteristics significantly differ, which provides a measure of effectiveness of the characteristics used. Furthermore, denoting as $\tilde{d}_{i}^{a}$ dominating components of subsequent principal directions corresponding to averaged damage index $A D I_{a}$, one can consider effective averaged damage indices (eADI's) as:

$$
e A D I_{i}=\sum_{a} \tilde{d}_{i}^{a} A D I_{a}
$$

Since eADI's correspond to single signal characteristic they are easier to interpret compared to principal components $\lambda_{i}$ while still preserving properties of data separation.

Some of the proposed signal characteristics are defined by linear signal transformations so some of the effective averaged damage indices can be highly correlated. In this case they correspond to the same signal characteristic but with different weights $\tilde{d}_{i}^{a}$ assigned to signal transformation, e.g. Fourier filtering. Observations distorted by noise or originated from faulty generators resulting in particular in a different spectrum of the received signal are outlying from the correlation line and therefore can be dropped out providing a sensor network self diagnostic tool.

The most effective uncorrelated eADI's can be used to develop parametric (e.g. LDA, QDA, Bayesian) or nonparametric (k-nn, SVM) classification [19] as well as regression models for damage size estimation.

\subsection{SHM model verification}

The SHM system scheme presented in the previous section was verified on retrofitted aircraft structures [12], therefore certain restrictions on the sensor network configuration had to be taken into account. Two different types of specimens were prepared and fatigue tests were performed.

In order to increase the size of the trial, the following partially averaged damage indices (pADI's):

$$
p A D I_{j}(g)=\frac{1}{(S-1)} \sum_{\substack{s: \\ s \neq g}} D I_{j}(g, s)
$$

were used and their effective counterparts (epADI's) were defined analogously as in the previous section. 


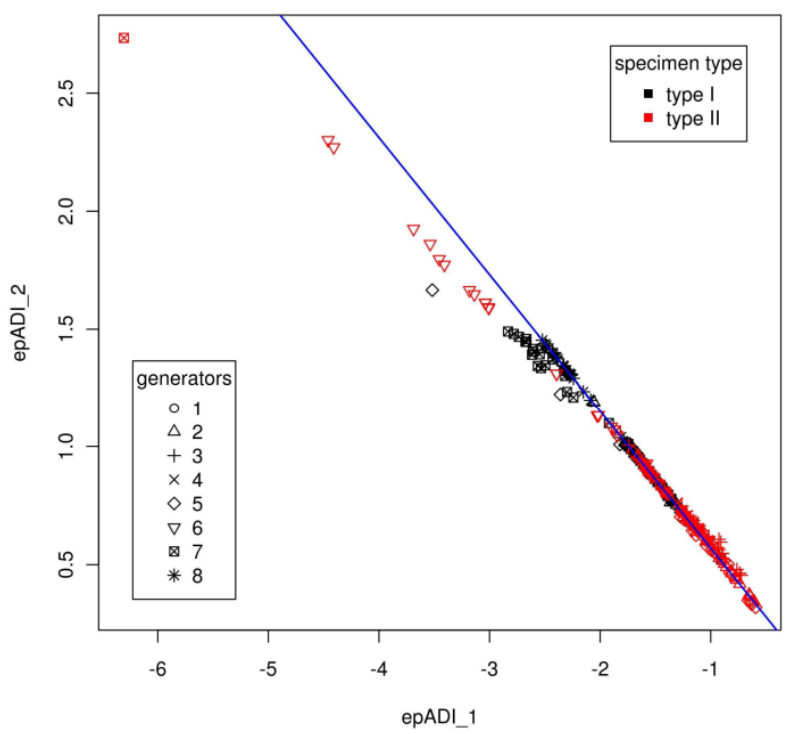

(a)

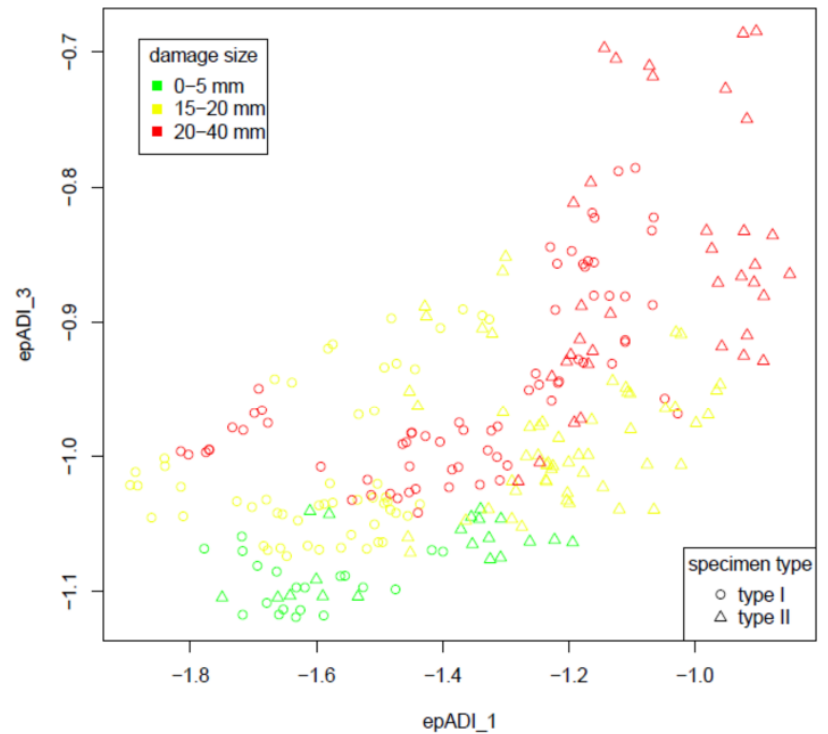

(b)

Fig. 1. Effective partially averaged damage indices for both specimens. (a) correlated epADI's (b) uncorrelated epADI's

The correlated effective pADI's were used to provide a network self diagnostic tool. Observations originated from generator no. 7 for type I specimen as well as single excitations from generator no. 3 and 5 are outlying from the correlation line and therefore were dropped out (Fig. 1(a)). Separation of the two most effective uncorrelated pADI's is presented on the figure (Fig. 1(b)). Since the interaction of elastic waves with a structure discontinuity is a local phenomenon, pADI's values depend strongly on the localization of a generator, e.g. its distance from damage. This is clearly visible on theplot (Fig. 1(b)), however data are separated for individual generators for both types of specimens (figure not shown here).

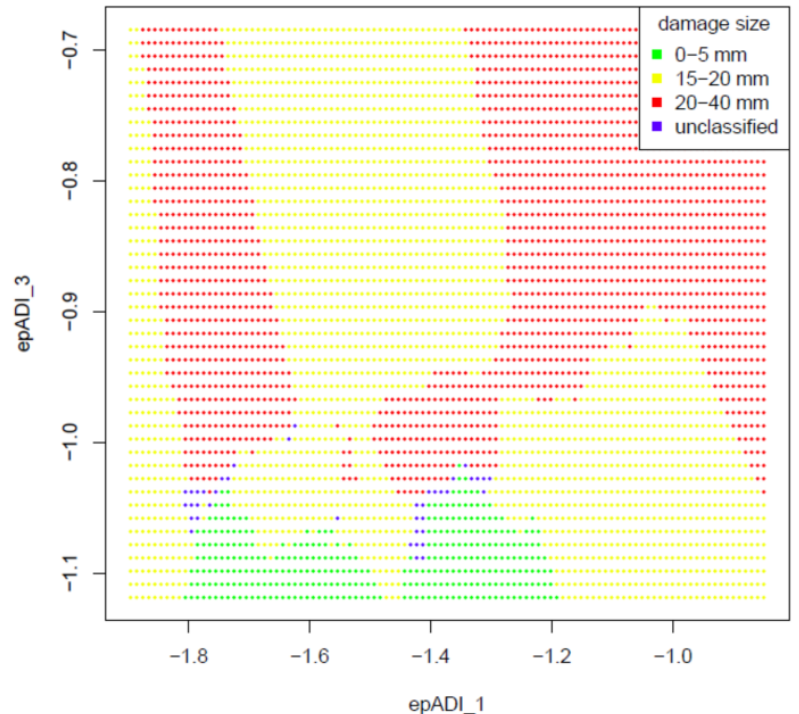

(a)
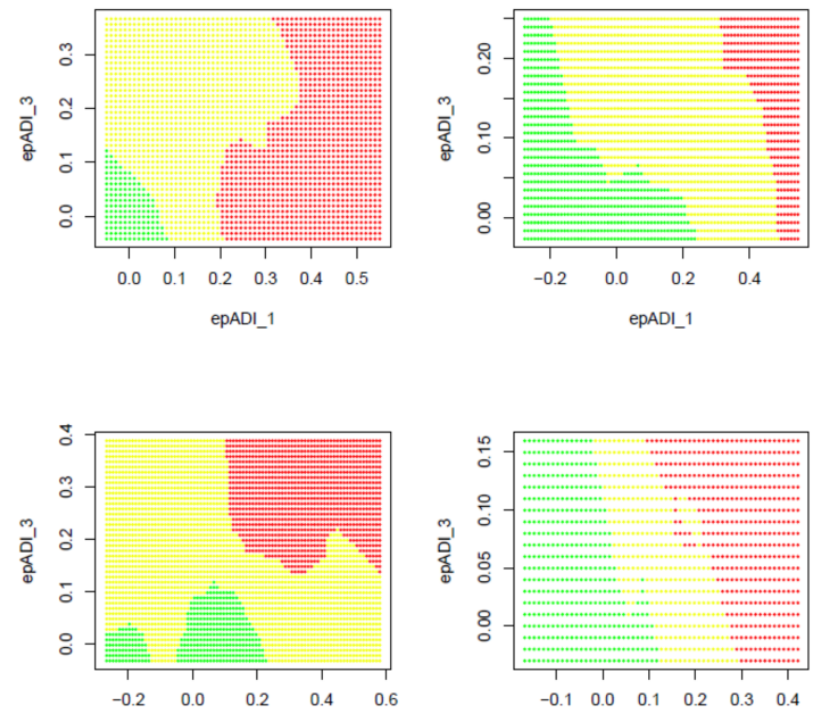

(b)

Fig. 2. Classification regions of nearest neighbor model. (a) direct 5-nn model, (b) 2-fold classification model 
These pADI's were used to provide two $k$ nearest neighbors (k-nn) models for damage size estimation. Classification regions for nearest neighbor models are calculated determining the most frequent class of $k$ samples from the training dataset that are the nearest to the given region point. Classification regions for the 5-nn model based on euclidean metric in the space spanned by two the most effective uncorrelated epADI's are presented in the Figure 2. In order to estimate the sufficient size of the learning dataset and model effeciency, a 5-fold cross-validation method [19] was utilised (Tab. 1). Due to pADI's dependence on the localization of the generator there occurs significant risk of type I misclassification.

Table 1. Cross-validation results of direct 5-nn model.

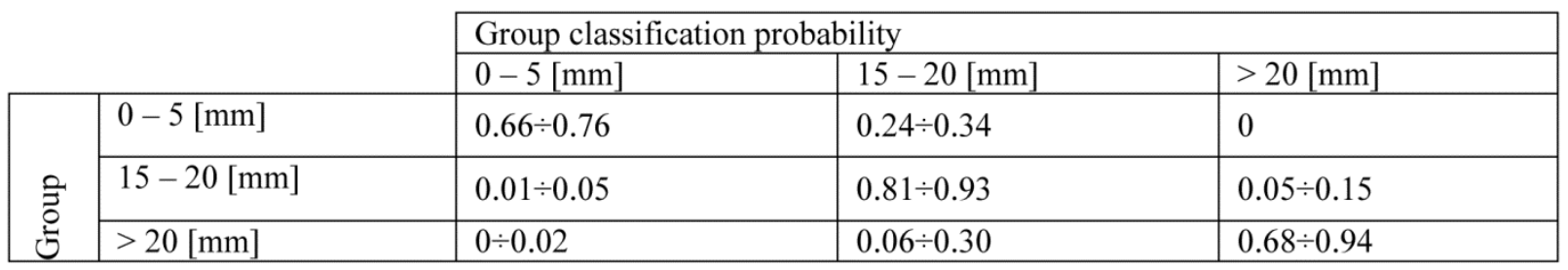

In order to avoid overlappping of classification regions for different generators and enhance system reliability a 2-fold classification model was developed. In this scheme the structural health assessment was performed based on a majority of indications from 3-nn models provided for each generator separately (Fig. 2(b)). This model was verified with use of leave-one-out crossvalidation [19] (Tab. 3). Since classification regions of undamaged (0-5 [mm]) and seriously damaged (20$40[\mathrm{~mm}]$ ) specimens for this model are separated (Fig. 2(b)) there is virtually no risk of type I misclassification (Tab. 2) compared to direct the 5-nn model (Tab. 1).

Table 2. Cross-validation results of the 2-fold classification model.

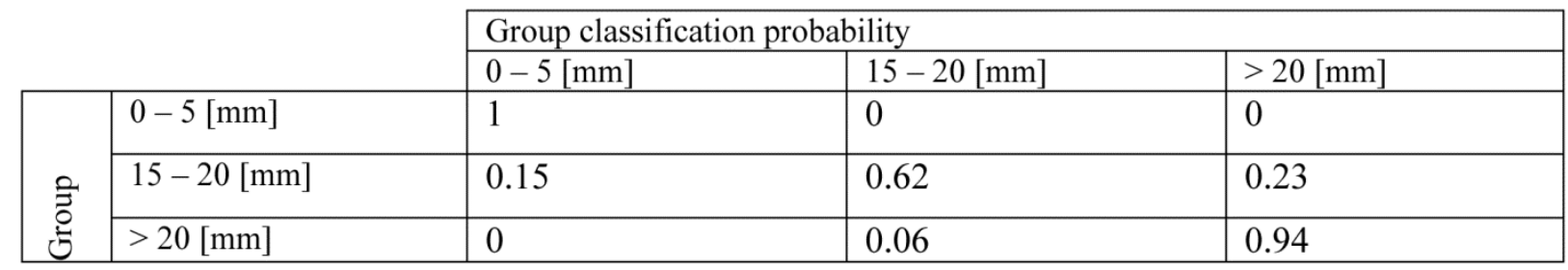

\section{CONCLUSIONS}

In this paper a SHM model meeting certain requirements of the SHM paradigm was presented and verified using real aircraft structures. The main challenge before implementing the system into operational phase is to assure its durability and reliability, e.g. the possibility of replacement of faulty PZT transducers in the sensor network. This will require developing the quasi baseline free methods of inference in order to eliminate the necessity of calibrating the functioning of a given network cell after the sensor replacement. However, training dataset will be needed in the system design phase. The proposed signal characteristics should be robust enough to provide such quantitative structural health assessment after signal denoising and other signal transformations.

The financial support from the National Centre for Research and Development for the work realized under the LIDER project LIDER/25/43/L-2/10/NCBiR/2011 is gratefully acknowledged. 


\section{REFERENCES}

[1] Worden, K. \& Barton, J.M. (2004). An Overview of Intelligent Fault Detection in Systems and Structures. Structural Health Monitoring. Vol. 3(1), p. 85.

[2] Medina, E.A. \& Aldrin, J.C. (2011). Probabilistic Reliability Assessment Protocol for Structural Health Monitoring (SHM) Systems. In AA\&S 2011 Conference, April 18-21 2011, San Diego.

[3] Su, Z. \& Ye, L. (2009). Identification of Damage Using Lamb Waves. London: SpringerVerlag GmbH \& Co.

[4] Alleyne, D.N. \& Cawley, P. (1996). The excitation of Lamb waves in pipes using drycoupled piezoelectric transducers. J. Nondestruct. Eval. Vol. 15(1), p. 11.

[5] Tua, P.S., Quek, S.T. \& Wang, Q. (2005). Detection of cracks in cylindrical pipes and plates using piezo-actuated Lamb waves. Smart Mater. Struct. Vol. 14, p. 1325.

[6] Kim Y.H., Kim D.H., Han J.H. \& Kim C.G. (2007). Damage assessment in layered composites using spectral analysis and Lamb wave. Compos. Part B-Eng. Vol. 38, p. 800.

[7] Su, Z., Ye, L. \& Lu, Y. (2006). Guided Lamb waves for identification of damage in composite structures: a review. J. Sound Vib. Vol. 295, p. 753.

[8] Wang, Q.W. \& Sun, B.X. (2010). Structural damage localization and quantification using static test data. Struct. Health Monit. Vol. 10(4), p. 381.

[9] Hay T.R., Royer R.L., Gao H. , Zhao X. \& Rose J.L. (2006). A comparison of embedded sensor Lamb wave ultrasonic tomography approaches for material loss detection. Smart Mater. Struct. Vol. 15(4), p. 946.

[10] Wang D., Ye L., Lu Y. \& Su Z. (2009). Probability of the presence of damage estimated from an active sensor network in a composite panel of multiple stiffeners. Compos. Sci. Technol. Vol. 69(13), p. 2054.

[11] Clarke, T. \& Cawley, P. (2010). Enhancing the defect localization capability of a guided wave SHM system applied to a complex structure. Struct. Health Monit. Vol. 10(3), p. 247.

[12] Dragan K., Klimaszewski S., Salacinski M., Synaszko P. \& Dziendzikowski M. (2011), Structural Health Monitoring of the Helicopter Main Rotor. In AA\&S 2011 Conference, April 18-21 2011, San Diego.

[13] Dragan K., Dziendzikowski M. \& Uhl T. (2011). The development of the non-parametric classification models for the damage monitoring on the ex ample of the ORLIK aircraft structure. In Second International Workshope on Smart Diagnostic of Structures, Krakow, November 2011.

[14] Sohn, H., Czarnecki, J.A. \& Farrar, C.R. (2000). Structural health monitoring using statistical process control. J. Struct. Eng. Vol. 126(1), p. 1356.

[15] De Boe, P. \& Golinval, J.C. (2003). Principal component analysis of a piezosensor array for damage localization. Struct. Health Monit. Vol. 2(2), p. 137.

[16] Mustapha F., Worden K., Pierce S.G. \& Manson G. (2007). Damage detection using stress waves and multivariate statistics: an experimental case study of an aircraft component. Strain, Vol. 43(1), p. 47.

[17] Trendafilova, I., Cartmell, M.P. and Ostachowicz, W. (2008). Vibration-based damage detection in an aircraft wing scaled model using principal component analysis and pattern recognition. J. Sound Vib. Vol. 313(3), p. 560.

[18] Mujica L.E., Rodellar J., Fernandez A. \& Guemes A. (2010). Q-statistic and T2 statistic PCA-based measures for damage assessment in structures. Struct. Health Monit. Vol. 10(5), p. 539.

[19] Hastie, T. Tibshirani, R. \& Friedman, J. (2009). The Elements of Staistical Learning: Data Mining, Inference, and Prediction, second ed. New York: Springer Science+Business Media. 